\title{
Role of Methylcitric Acid Cycle in Catabolism of Amino Acids by Saccharomyçopsis lipolytica
}

\author{
Shunichi Miyakoshi, Kengo Enami, Hiroo Uchiyama* \\ and Takeshi TABUCHI \\ Institute of Applied Biochemistry, University of Tsukuba, \\ Sakura-mura, Ibaraki 305, Japan \\ * National Institute for Environmental Studies, \\ Yatabe-machi, Ibaraki 305, Japan \\ Received September 8, 1986
}

\begin{abstract}
We examined the production of 2-methylisocitric acid, an intermediate of the constitutive methylcitric acid cycle involved in propionyl-CoA oxidation during the catabolism of different amino acids by a mutant lacking 2-methylisocitrate lyase, a key enzyme of the cycle. The acid was produced equimolarly from isoleucine within a range of amounts of the amino acid added. The amount of the acid produced also increased depending upon the amounts of valine, threonine, methionine, homoserine, and $\alpha$-aminobutyric acid. However, only a little acid was produced from the 13 other amino acids tested. These results indicated that propionyl-CoA was involved in the catabolism of the first six amino acids named, but not in the other 13. Intracellular amino acids were therefore part of metabolic turnover and the constitutive cycle functioned in the catabolism of propionyl-CoA derived from the turnover.
\end{abstract}

Many yeasts produce citric and isocitric acids from various carbon sources, including n-alkanes. ${ }^{1)}$ A mutant of Saccharomycopsis lipolytica has been found to produce threo- $-\mathrm{D}_{\mathrm{s}}$ 2-methylisocitric acid (MICA) in large amounts from odd-numbered $n$-alkans. ${ }^{2}$ From this finding and other observations, we have proposed that there is a methylcitric acid cycle involved in the oxidation of propionyl-CoA into pyruvate. ${ }^{3,4)}$ The mutant has completely lost 2-methylisocitrate lyase (EC 4.1.3.30), a key enzyme of the cycle. ${ }^{5)}$ The cycle enzymes are constitutive in $S$. lipolytica. ${ }^{6,7)}$ Propionate normally arises from the catabolism of branched aliphatic amino acids such as isoleucine and valine in bacteria and mammals. ${ }^{8,9)}$ Here, we studied the significance of this constitutive cycle in the catabolism of amino acids by $S$. lipolytica. The mutant produced MICA abundantly from several amino acids.

\section{MATERIALS AND METHODS}

Microorganism. S. lipolytica No. R-2 used was derived from strain IFO $1659 .{ }^{10)}$ This mutant, which has lost 2 methylisocitrate lyase, ${ }^{5)}$ is an excellent producer of MICA from odd-numbered $n$-alkanes. ${ }^{2)}$

Media and culture conditions. The seed culture medium contained $8 \%$ glucose, $0.3 \% \quad \mathrm{NH}_{4} \mathrm{Cl}, 0.05 \% \quad \mathrm{KH}_{2} \mathrm{PO}_{4}$, $0.05 \% \mathrm{MgSO}_{4} \cdot 7 \mathrm{H}_{2} \mathrm{O}, 10 \mu \mathrm{g} /$ liter thiamine- $\mathrm{HCl}$, and $1 \%$ $\mathrm{CaCO}_{3}$ (sterilized separately) in tap water. The basal fermentation medium contained glucose or $n$-hexadecane as the carbon source, $\mathrm{NH}_{4} \mathrm{Cl}$ or amino acids as the nitrogen source, $0.01 \% \mathrm{NaH}_{2} \mathrm{PO}_{4}, 0.2 \% \mathrm{KCl}, 0.05 \%$ $\mathrm{MgSO}_{4} \cdot 7 \mathrm{H}_{2} \mathrm{O}, 50 \mu \mathrm{g} /$ liter thiamine- $\mathrm{HCl}$ and $7.5 \% \mathrm{CaCO}_{3}$ (sterilized separately) in tap water. The carbon and the nitrogen sources used and their concentrations are described in legends of the figures. A seed culture was prepared by inoculation of cells grown on potato-glucose agar slants into $300-\mathrm{ml}$ Erlenmeyer flasks containing $30 \mathrm{ml}$ of the seed culture medium, followed by incubation at $26^{\circ} \mathrm{C}$ for 2 days on a rotary shaker $(220 \mathrm{rpm})$. The seed culture $(2 \mathrm{ml})$ was transferred to $500-\mathrm{ml}$ Erlenmeyer flasks containing $40 \mathrm{ml}$ of the basal fermentation medium. The flasks were shaken on a rotary shaker at $26^{\circ} \mathrm{C}$ for 5 days in glucose medium and for 8 days in $n$-hexadecane medium. 
Analytical method. The amounts of organic acids in culture broths were assayed by gas chromatography under the conditions described previously. ${ }^{2)}$

Chemicals. $n$-Hexadecane, purchased from Tokyo Kasei Kogyo Co., Ltd., was certified to be $98 \%$ pure. All amino acids were purchased from Wako Pure Chemical Industries, Ltd., and were extra pure grade and $\mathrm{L}$-form, except for DL- $\alpha$-amino- $n$-butyric acid and DL-homoserine.

\section{RESULTS}

\section{Production of 2-methylisocitric acid in glucose medium}

The production of MICA from some amino acids by the mutant was examined in the basal fermentation medium containing $10 \%$ glucose as the major carbon source and $50 \mathrm{mM} \mathrm{NH}_{4} \mathrm{Cl}$ or one of the amino acids as the sole nitrogen source. MICA was produced in high concentrations in media supplied with valine, isoleucine, threonine, or methionine (Fig. 1). MICA was produced in low concentrations (about 2 to $3 \mathrm{~mm}$ ) in media supplied with $\mathrm{NH}_{4} \mathrm{Cl}$, aspartic acid, or lysine. The ability of the mutant to degrade individual amino acids as the sole nitrogen source was found to differ by comparison of the different amounts of citric and isocitric acids produced.

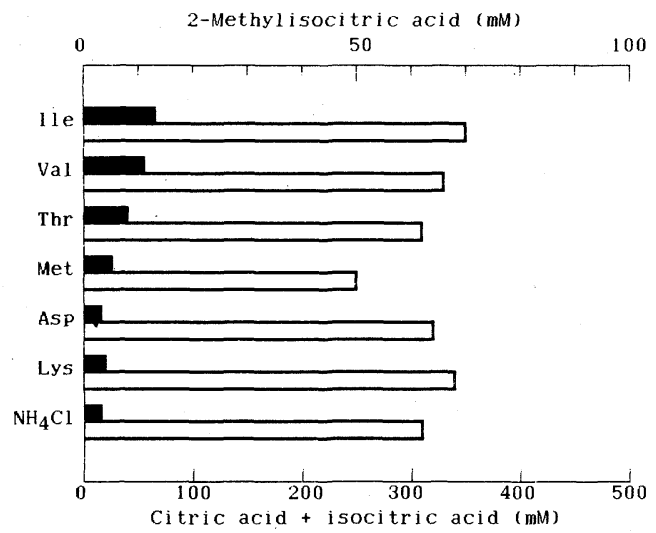

FIG. 1. Production of 2-Methylisocitric Acid from Amino Acids in Glucose Media.

The fermentation medium contained $10 \%$ glucose and $50 \mathrm{mM} \mathrm{NH} \mathrm{N}_{4} \mathrm{Cl}$ or the amino acid listed at the left of the figure. $\square$, 2-methylisocitric acid; $\square$, citric acid + isocitric acid.
Production of 2-methylisocitric acid from different amino acids in n-hexadecane media

Differences in the amounts of MICA produced in glucose media were not as large as we expected, probably because of partial repression of the synthesis of the enzymes in the cycle. $^{6)}$

The production of MICA from 20 amino acids was examined in basal fermentation media containing $7.5 \%(\mathrm{v} / \mathrm{v}) n$-hexadecane instead of glucose as the major carbon source

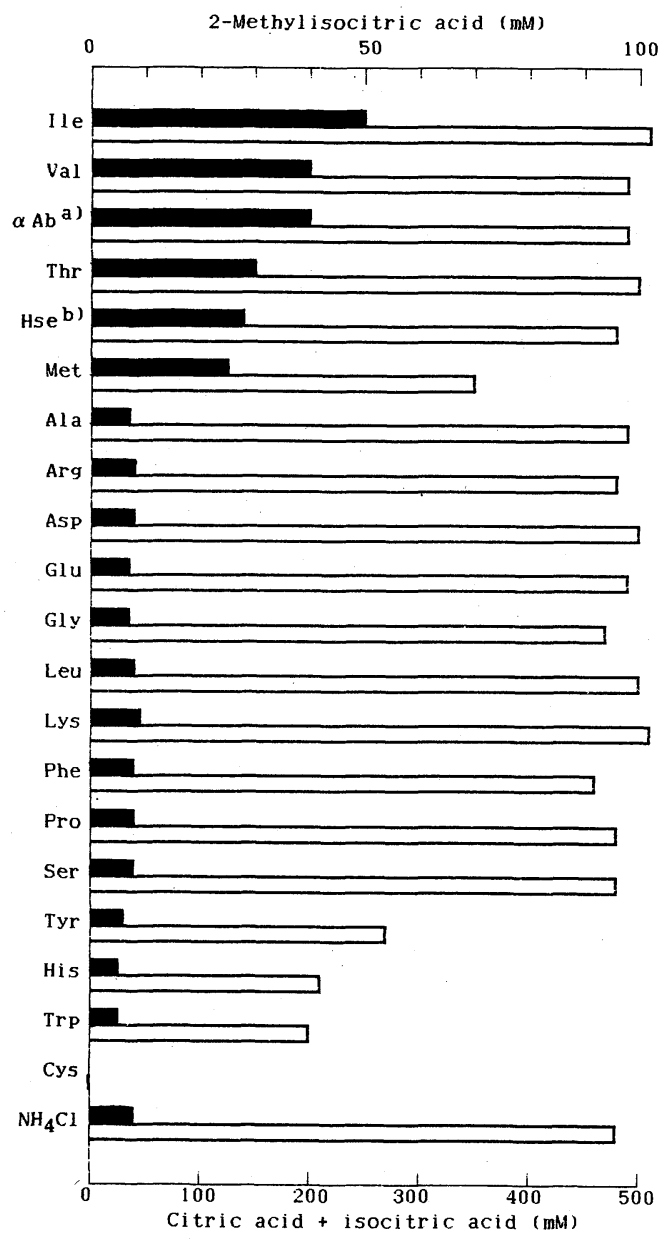

FIG. 2. Production of 2-Methylisocitric Acid from Amino Acids in $n$-Hexadecane Media.

The fermentation medium contained $7.5 \%$ (v/v) $n$ hexadecane, $0.05 \%$ Span 80 , and $50 \mathrm{~mm} \mathrm{NH}_{4} \mathrm{Cl}$ or the amino acid listed at the left of the figure. $\square, 2-$ methylisocitric acid; $\square$, citric acid + isocitric acid.

a DL- $\alpha$-Amino- $n$-butyric acid.

b DL-Homoserine. 


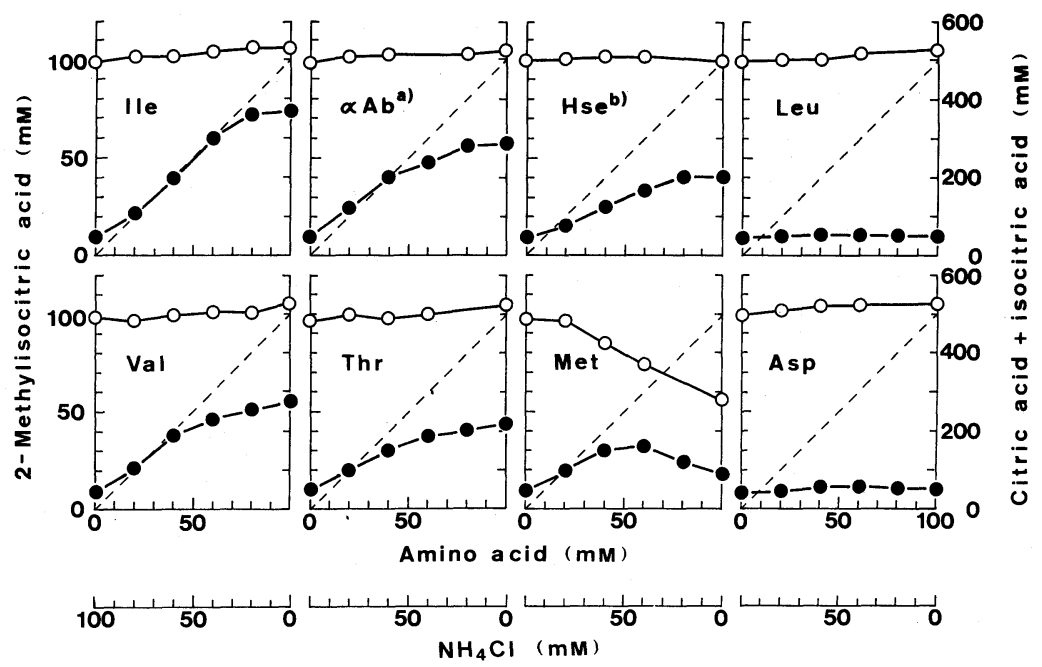

FIG. 3. Effects of Concentrations of Amino Acids on Production of 2-Methylisocitric Acid in $n$-Hexadecane Media.

The fermentation media contained $7.5 \%(\mathrm{v} / \mathrm{v}) n$-hexadecane, $0.05 \%$ Span 80 , and $\mathrm{NH}_{4} \mathrm{Cl}$ plus the amino acid at the total concentration of $100 \mathrm{~mm}$ as shown in the bottom of the figure. Dotted lines represent the equimolar relationship between concentrations of amino acids supplied and those of MICA produced.

methylisocitric acid; $\bigcirc-\mathrm{O}$, citric acid +isocitric acid.

${ }^{a}$ DL- $\alpha$-Amino- $n$-butyric acid.

b DL-Homoserine.

and one of the amino acids as a minor carbon and sole nitrogen source. The mutant used all of the amino acids except cysteine as sole nitrogen sources, and citric and isocitric acids were produced abundantly in media supplied with the individual amino acids except for tryptophan, histidine, tyrosine, and methionine (Fig. 2).

MICA was excreted in appreciable high concentrations (about 25 to $50 \mathrm{~mm}$ ) in media supplied with valine, isoleucine, threonine, methionine, homoserine, or $\alpha$-amino- $n$-butyric acid as the nitrogen source. MICA was produced in low concentrations ( 5 to $8 \mathrm{~mm}$ ) in media supplied with $\mathrm{NH}_{4} \mathrm{Cl}$ or the 13 other amino acids.

Effects of concentrations of different amino acids on production of 2-methylisocitric acid

A high concentration of the nitrogen source led to a low yield of citric acid in a medium supplied with $0.05 \% \mathrm{KH}_{2} \mathrm{PO}_{4}$, as reported before. ${ }^{11)}$ Medium composition with an excess of total nitrogen but with little effect on acid production was first screened. Preliminary experiments showed that an excess of the nitrogen source, $100 \mathrm{mM} \mathrm{NH} \mathrm{NH}_{4} \mathrm{Cl}$, did not affect acid production in the basal fermentation medium supplied with a low concentration of phosphate $\left(0.01 \% \mathrm{NaH}_{2} \mathrm{PO}_{4}\right)$ and with a high concentration of potassium salt $(0.2 \% \mathrm{KCl})$ instead of $0.05 \% \mathrm{KH}_{2} \mathrm{PO}_{4}$.

Figure 3 shows the effects of concentrations of the six amino acids from which MICA was produced in high yields on acid production with an excess concentration of $100 \mathrm{~mm}$ for the total nitrogen source. MICA was produced almost equimolarly up to $60 \mathrm{~mm}$ from isoleucine. From the five other amino acids, the amounts of MICA produced also increased to some extent depending upon the amounts of the amino acid added. These results indicated that the six amino acids were catabolized even in the presence of an excess of $\mathrm{NH}_{4} \mathrm{Cl}$, and that the amounts of MICA produced depended on the amounts of propionyl-CoA available from the oxidative degradation of these amino acids. 
Figure 3 also shows the effects of concentrations of leucine and aspartic acid, from which MICA was produced in low yields on acid production at a constant concentration for the total nitrogen source. MICA was produced at the concentrations of about $8 \mathrm{~mm}$ regardless of the concentrations of the amino acids supplied. These results indicated that propionyl-CoA was not involved in the catabolism of leucine or aspartic acid. Glutamic acid and lysine also gave similar results.

\section{DISCUSSION}

The methylcitric acid cycle is involved not in the anabolic reaction sequence related to propionate but in the purely catabolic reaction sequence of propionyl-CoA to pyruvate, because the mutant, which completely lacks 2methylisocitrate lyase, grows well on ordinary synthetic media, like its parent.

In alcohol fermentation, fusel oils are produced from amino acids, but little is known about the aerobic degradation of amino acids yielding propionyl-CoA by yeasts. Since the mutant can excrete MICA as the detoxified product of propionate, we can infer the participation of propionyl-CoA during the catabolism of amino acids from the excretion of MICA by the mutant. The mutant produced MICA abundantly from six amino acids. These results show that propionyl-CoA is involved in the catabolism of these amino acids, but not with that of the 13 other amino acids tested.

Figure 4 summarized these results and information from the literature. ${ }^{9,12)}$ Three pathways for the catabolism of threonine are known. ${ }^{12)}$ One pathway involves conversion to 2-oxobutyrate, ${ }^{13)}$ a second one involves cleavage into acetaldehyde and glycine, and a third one involves dehydration to 2-amino-3-oxobutyrate, followed by conversion to aminoacetone $^{14)}$ or by cleavage into acetyl-CoA and glycine. ${ }^{15)}$ Because of the participation of propionyl-CoA, S. lipolytica must catabolize threonine through the first pathway (Fig. 4).

MICA was produced in concentrations of

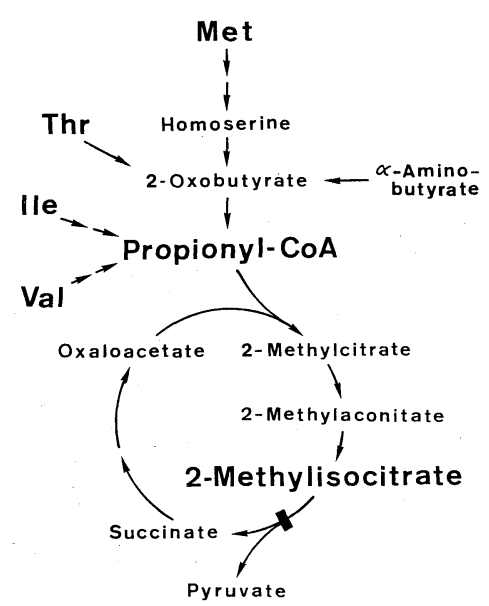

Fig. 4. Presumptive Pathways Yielding Propionyl-CoA during Catabolism of Amino Acids by Saccharomycopsis lipolytica.

None of the other amino acids listed in Fig. 2 will yield propionyl-CoA during their catabolism. $\square$, the reaction that is blocked in the mutant.

about $2 \mathrm{~mm}$ even from the synthetic medium containing glucose as the carbon source and $\mathrm{NH}_{4} \mathrm{Cl}$ as the nitrogen source. The cycle enzymes were constitutively synthesized in the parent cells grown on a similar glucose medium described elsewhere. ${ }^{6)}$ The accumulation of MICA in the glucose medium can be explained if endogenous amino acids are subject to metabolic turnover in growing cells of $S$. lipolytica. That is, propionyl-CoA is continually formed as an intermediate of the turnover of several amino acids. Accordingly, the cycle enzymes are constitutively required for the catabolism of propionyl-CoA.

\section{REFERENCES}

1) T. Tabuchi, M. Tanaka, Y. Tahara and M. Abe, Nippon Nōgeikagaku Kaishi, 44, 562 (1970).

2) T. Tabuchi and N. Serizawa, Agric. Biol. Chem., 39, 1049 (1975).

3) T. Tabuchi and N. Serizawa, Agric. Biol. Chem., 39, 1055 (1975).

4) T. Tabuchi, H. Aoki, H. Uchiyama and T. Nakahara, Agric. Biol. Chem., 45, 2823 (1981).

5) T. Tabuchi and T. Satoh, Agric. Biol. Chem., 40, 1863 (1976).

6) T. Tabuchi and K. Igoshi, Agric. Biol. Chem., 42, 2381 (1978). 
7) H. Uchiyama, M. Ando, Y. Toyonaka and T. Tabuchi, Eur. J. Biochem., 125, 523 (1982).

8) Y. Kaziro and S. Ochoa, Adv. Enzymol., 26, 283 (1964).

9) L. K. Massey, J. R. Sokatch and R. S. Conrad, Bacteriol. Rev., 40, 42 (1976).

10) T. Tabuchi and S. Hara, Agric. Biol. Chem., 38, 1105 (1974).

11) T. Tabuchi, Nippon Nōgeikagaku Kaishi, 47, 479
(1973).

12) "Metabolic Pathways," Vol. 3, 3rd Ed., ed. by D. M. Greenberg, Academic Press, New York, 1969, p. 122.

13) M. Tokushige and O. Hayaishi, J. Biochem., 72, 471 (1972).

14) A. Neuberger and G. H. Tait, Biochim. Biophys. Acta, 41, 164 (1960).

15) S. C. Bell and J. M. Turner, Biochem. J., 156, 449 (1976). 